\title{
Robust exponential attractors for a parabolic-hyperbolic phase-field system
}

\author{
Cyril D. Enyi ${ }^{1 *}$
}

\section{"Correspondence:}

enyicyrildennis@gmail.com

${ }^{1}$ College of Sciences, Department of

Mathematics, University of Hafr Al

Batin, Hafr Al Batin, Saudi Arabia

\section{Springer}

\begin{abstract}
In this paper, we construct a robust family of exponential attractors for a parabolic-hyperbolic phase-field system (PHPFS), which describes phase separation in material sciences, e.g., melting and solidification. A consequence of this is the existence of finite fractal dimensional global attractors which are both upper and lower semicontinuous at the parameter $\epsilon=0$. Hence we establish the convergence of the dynamics of PHPFS to those of the well known Cagilnap phase-field system.
\end{abstract}

MSC: 35B41;81Q15; 82B26; 35M33

Keywords: Parabolic-hyperbolic phase-field system; Exponential attractors; Continuity

\section{Introduction}

Exponential attractors are compact and positively invariant sets with finite fractal dimension which attract all the trajectories starting from bounded sets at a uniform exponential rate (see $[5-7,14])$. The existence of exponential attractors guarantees the existence of a finite fractal dimensional global attractor. Readers may see $[4,8,13]$ and references therein for more on the dimension of a global attractor. Thus a finite-dimensional reduction principle can be applied to reduce the infinite-dimensional dynamical system under consideration to a finite-dimensional system of ODEs. The sensitivity of exponential attractors under small perturbations is the main focus in this work. One may see [15] for some recent developments in the construction of exponential attractors.

The phase-field system is a system of equations which couples the temperature $u$ and order parameter $\phi$ also known as "phase-field". It describes phase separations in materials occupying a domain $\Omega \subset \mathbb{R}^{d}$.

We consider the following parabolic-hyperbolic phase-field system (PHPFS):

$$
\left\{\begin{array}{l}
\epsilon \phi_{t t}+\phi_{t}-\Delta \phi+\phi+g(\phi)-u=0 \\
u_{t}+\phi_{t}-\Delta u=0 \\
\left.\partial_{n} \phi\right|_{\partial \Omega}=\left.u\right|_{\partial \Omega}=0 \\
\phi(0)=\phi_{0}, \phi_{t}(0)=\phi_{1}, u(0)=u_{0}
\end{array}\right.
$$

in a bounded domain $\Omega \subset \mathbb{R}^{d}, d=1,2,3$ with smooth boundary $\partial \Omega$, where $\epsilon \in(0,1]$ is a small parameter. Denote the function $G(s)=\int_{0}^{s} g(\varsigma) d \varsigma$ and assume that $g$ satisfies $g \in$

(c) The Author(s) 2018. This article is distributed under the terms of the Creative Commons Attribution 4.0 International License (http://creativecommons.org/licenses/by/4.0/), which permits unrestricted use, distribution, and reproduction in any medium, provided you give appropriate credit to the original author(s) and the source, provide a link to the Creative Commons license, and indicate if changes were made. 
$\mathcal{C}^{2}(\mathbb{R})$ and the following conditions hold (cf., e.g., $\left.[1,3]\right)$ :

$$
\begin{aligned}
& G(s) \geq-C_{1}, \quad C_{1} \geq 0, \quad \forall s \in \mathbb{R}, \\
& \forall \gamma \in \mathbb{R}, \exists C_{2}(\gamma)>0, C_{3}(\gamma) \geq 0 \text { such that } \\
& (s-\gamma) g(s)-C_{2} G(s) \geq-C_{3}, \quad \forall s \in \mathbb{R}
\end{aligned}
$$

(where $C_{2}, C_{3}$ are bounded when $\gamma$ is bounded),

$$
\begin{aligned}
& g^{\prime}(s) \geq-C_{4}, \quad C_{4} \geq 0, \quad \forall s \in \mathbb{R}, \\
& \left|g^{\prime \prime}(s)\right| \leq C_{5}\left(|s|^{p}+1\right), \quad C_{5}>0, \quad \forall s \in \mathbb{R},
\end{aligned}
$$

with $p \geq 0$ when $d=1,2$ and $p \in[0,1]$ when $d=3$. We note that in space dimension one, no growth assumption on $g$ is needed.

We remark that our results also hold when $\phi$ is subject to a boundary condition of periodic type

$$
\left\{\begin{array}{l}
\left.u\right|_{x_{i}=0}=\left.u\right|_{x_{i}=L_{i}},\left.\quad u_{x_{i}}\right|_{x_{i}=0}=\left.u_{x_{i}}\right|_{x_{i}=L_{i}}, \quad i=1, \ldots, d \\
\left.\phi\right|_{x_{i}=0}=\left.\phi\right|_{x_{i}=L_{i}}, \quad i=1, \ldots, d, \\
\text { for } \phi \text { and the derivatives of } \phi \text { of order } \leq 3
\end{array}\right.
$$

if $\Omega=\prod_{i=1}^{d}\left(0, L_{i}\right)$.

We shall construct a robust family of exponential attractors which are both upper and lower semicontinuous at $\epsilon=0$ with respect to a norm independent of $\epsilon$.

Grasselli and Pata [10] showed a well-posedness result and the existence of the global attractor for the system $(\epsilon>0)$

$$
\left\{\begin{array}{l}
\epsilon \phi_{t t}+\phi_{t}-\Delta \phi+\phi^{3}=\gamma(\phi)+\lambda^{\prime}(\phi) u \\
u_{t}+\lambda^{\prime}(\phi) \phi_{t}-\Delta u=f
\end{array}\right.
$$

Grasselli and Pata [11] considered the system $(\epsilon>0)$

$$
\left\{\begin{array}{l}
\epsilon \phi_{t t}+\phi_{t}-\Delta \phi+\phi-\lambda^{\prime}(\phi) u+h(\phi)=\xi \\
u_{t}+\lambda^{\prime}(\phi) \phi_{t}-\Delta u=0
\end{array}\right.
$$

in 3D, subject to mixed boundary conditions, Neumann on $\phi$ and Dirichlet on $u$. They proved a well-posedness result, the existence of the global attractor and its upper semicontinuity at $\epsilon=0$, and constructed exponential attractors with respect to a norm depending on $\epsilon$. Also, Grasselli et al. [9] gave a well-posedness result and constructed a robust family of exponential attractors $\mathbb{E}_{\epsilon}$ for the system

$$
\left\{\begin{array}{l}
\epsilon \phi_{t t}+\phi_{t}-\Delta \phi-\lambda^{\prime}(\phi) u+\chi(\phi)=\xi \\
u_{t}+\lambda^{\prime}(\phi) \phi_{t}-\Delta u=0
\end{array}\right.
$$

in 3D, subject to Dirichlet boundary conditions on both $\phi$ and $u$, where $\chi(\phi)$ is singular at $\phi= \pm 1$, e.g., $\ln \left(\frac{1+\phi}{1-\phi}\right), \phi \in(0,1)$. More precisely, they showed that there exist $c>0$ and 
$\varpi \in(0,1)$, both independent of $\epsilon$, such that

$$
\operatorname{dist}_{K, \epsilon}^{\mathrm{sym}}\left(\mathbb{E}_{\epsilon}, \mathbb{E}_{0}\right) \leq c \epsilon^{\sigma}, \quad \forall \epsilon \in[0,1]
$$

in the norm $\left\|\left(\phi, \phi_{t}, u\right)\right\|_{K, \epsilon}^{2}=\|\Delta \phi\|_{L^{2}(\Omega)}^{2}+\epsilon\left\|\nabla \phi_{t}\right\|_{L^{2}(\Omega)}^{2}+\left\|\phi_{t}\right\|_{L^{2}(\Omega)}^{2}+\|\Delta u\|_{L^{2}(\Omega)}^{2}$, which clearly depends on $\epsilon$.

Finally, we would also like to mention the papers $[12,16,17]$ where the convergence to equilibrium of solutions for a parabolic-hyperbolic phase-field model were proven.

This work is organized as follows. In Sect. 1, we give a brief introduction. In Sect. 2, we give some a priori estimates. In Sect. 3, we construct exponential attractors for the system (1.1). Finally, in Sect. 4, we construct a robust family of exponential attractors which are both upper and lower semicontinuous at $\epsilon=0$ for the system (1.1).

We define the Hilbert space $\mathcal{H}_{r, \epsilon}=H^{r} \times H^{r-1} \times H_{0}^{r-1}, r \geq 1$, endowed with the norm

$$
\|(\varphi, \psi, v)\|_{\mathcal{H}_{r, \epsilon}}=\left(\|\varphi\|_{r}^{2}+\epsilon\|\psi\|_{r-1}^{2}+\|v\|_{r-1}^{2}\right)^{1 / 2}
$$

where we understand that $H_{0}^{0}=H^{0}(\Omega)=L^{2}(\Omega)$. Hence, we denote $\mathcal{H}_{1,0}=H^{1}(\Omega) \times L^{2}(\Omega)$, endowed with the norm $\|(\cdot, \cdot)\|_{\mathcal{H}_{1,0}}=\left(\|\cdot\|_{1}^{2}+\|\cdot\|^{2}\right)^{1 / 2}$.

\section{A priori estimates}

We multiply $(1.1)_{1}$ by $\phi_{t}$ and $(1.1)_{2}$ by $u$, then integrate over $\Omega$. Adding the resulting equations, we obtain

$$
\frac{d}{d t} E_{1}(t)+2\left\|\phi_{t}\right\|^{2}+2\|\nabla u\|^{2}=0
$$

where

$$
E_{1}(t)=\|\nabla \phi\|^{2}+\|\phi\|^{2}+\epsilon\left\|\phi_{t}\right\|^{2}+\|u\|^{2}+2 \int_{\Omega} G(\phi) d x .
$$

From (1.2), (1.3) and (1.5), we deduce that

$$
\int_{\Omega} G(\phi) d x \geq-C_{1}|\Omega| \quad \text { and } \quad \int_{\Omega} G(\phi) d x \leq c\left(\|\phi\|_{1}^{p+3}+1\right) .
$$

Hence,

$$
\left\|\left(\phi, \phi_{t}, u\right)\right\|_{\mathcal{H}_{1, \epsilon}}^{2}-\alpha_{1} \leq E_{1}(t) \leq \alpha_{2}\left(\|\phi\|_{1}^{p+3}+\epsilon\left\|\phi_{t}\right\|^{2}+\|u\|^{2}+1\right),
$$

for some $\alpha_{1}, \alpha_{2}>0$ independent of $\epsilon$. Thus integrating (2.1) over $(0, t)$ and accounting for (2.2), we obtain that

$$
\int_{0}^{t}\left(\left\|\phi_{t}(s)\right\|^{2}+\|\nabla u(s)\|^{2}\right) d s \leq E_{1}(0)+\alpha_{1}, \quad \forall t \geq 0 .
$$

Hence by (2.2) again, we get

$$
\int_{0}^{\infty}\left(\left\|\phi_{t}(s)\right\|^{2}+\|\nabla u(s)\|^{2}\right) d s \leq c\left(\left\|\phi_{0}\right\|_{1}^{p+3}+\epsilon\left\|\phi_{1}\right\|^{2}+\left\|u_{0}\right\|^{2}+1\right) .
$$


Let $\left(\phi^{1}, u^{1}\right)$ and $\left(\phi^{2}, u^{2}\right)$ be two solutions of (1.1). Set $\phi=\phi^{1}-\phi^{2}, \phi_{t}=\phi_{t}^{1}-\phi_{t}^{2}$ and $u=$ $u^{1}-u^{2}$, then $\phi(0)=0, \phi_{t}(0)=0$ and $u(0)=0$. The pair $\left(\phi, \phi_{t}, u\right)$ is a solution to the problem

$$
\left\{\begin{array}{l}
\epsilon \phi_{t t}+\phi_{t}-\Delta \phi+\phi+g\left(\phi^{1}\right)-g\left(\phi^{2}\right)-u=0 \\
u_{t}+\phi_{t}-\Delta u=0 \\
\phi(0)=\phi_{t}(0)=u(0)=0
\end{array}\right.
$$

We multiply (2.4) $)_{1}$ and $(2.4)_{2}$ by $\phi_{t}$ and $u$, respectively, integrate over $\Omega$, then add the resulting equations to get

$$
\frac{1}{2} \frac{d}{d t}\left(\|\nabla \phi\|^{2}+\|\phi\|^{2}+\epsilon\left\|\phi_{t}\right\|^{2}+\|u\|^{2}\right)+\left\|\phi_{t}\right\|^{2}+\|\nabla u\|^{2}=-\left(g\left(\phi^{1}\right)-g\left(\phi^{2}\right), \phi_{t}\right) .
$$

By Hölder's inequality and (1.5), we have

$$
\begin{aligned}
\left|\left(g\left(\phi^{1}\right)-g\left(\phi^{2}\right), \phi_{t}\right)\right| & \leq c\left(\left\|\phi^{1}\right\|_{L^{3 p+3}(\Omega)}^{p+1}+\left\|\phi^{2}\right\|_{L^{3 p+3}(\Omega)}^{p+1}+1\right)\|\phi\|_{L^{6}(\Omega)}\left\|\phi_{t}\right\| \\
& \leq c\left(\left\|\phi^{1}\right\|_{1}^{p+1}+\left\|\phi^{2}\right\|_{1}^{p+1}+1\right)\|\phi\|_{1}\left\|\phi_{t}\right\| .
\end{aligned}
$$

Therefore, by Young's inequality, we obtain

$$
\frac{d}{d t}\left(\|\nabla \phi\|^{2}+\|\phi\|^{2}+\epsilon\left\|\phi_{t}\right\|^{2}+\|u\|^{2}\right) \leq \tilde{M}(t)\|\phi\|_{1}^{2}
$$

where

$$
\tilde{M}(t)= \begin{cases}c \sup _{\theta \in[0,1]}\left\|g^{\prime}\left(\theta \phi_{1}+(1-\theta) \phi_{2}\right)\right\|_{L^{\infty}(\Omega)}^{2}, & \text { if } d=1, \\ c\left(\left\|\phi^{1}\right\|_{1}^{2 p+2}+\left\|\phi^{2}\right\|_{1}^{2 p+2}+1\right), & \text { if } d=2,3\end{cases}
$$

Noting that $t \mapsto \tilde{M}(t)$ is $L^{1}(0, T)$, and integrating (2.5) over $(0, t)$, we deduce that

$$
\left\|\left(\phi(t), \phi_{t}(t), u(t)\right)\right\|_{\mathcal{H}_{1, \epsilon}}^{2} \leq e^{\int_{0}^{t} \widetilde{M}(s) d s}\left\|\left(\phi(0), \phi_{t}(0), u(0)\right)\right\|_{\mathcal{H}_{1, \epsilon}}^{2}, \quad \forall t \geq 0 .
$$

We state a well-posedness result, which is proved in [11, Theorem 3.4].

Theorem 2.1 We assume that (1.2)-(1.5) hold. If $\left(\phi_{0}, \phi_{1}, u_{0}\right) \in \mathcal{H}_{1, \epsilon}$, then (1.1) possesses a unique solution $(\phi, u)$ such that

$$
\left(\phi, \phi_{t}, u\right) \in \mathcal{C}\left([0, T] ; \mathcal{H}_{1, \epsilon}\right)
$$

for any $T>0$. Moreover, if $\left(\phi_{0}, \phi_{1}, u_{0}\right) \in \mathcal{H}_{2, \epsilon}$, then $\left(\phi, \phi_{t}, u\right) \in \mathcal{C}\left([0, T] ; \mathcal{H}_{2, \epsilon}\right)$.

On account of Theorem 2.1 we can define the semigroup

$$
S_{\epsilon}(t): \mathcal{H}_{1, \epsilon} \rightarrow \mathcal{H}_{1, \epsilon}, \quad\left(\phi_{0}, \phi_{1}, u_{0}\right) \mapsto\left(\phi(t), \phi_{t}(t), u(t)\right), \quad \forall t \geq 0,
$$

where $\left(\phi(t), \phi_{t}(t), u(t)\right)$ is the solution to problem (1.1) at time $t$. The semigroup $S_{\epsilon}(t)$ is strongly continuous (cf. (2.6)). 
It is also known from [11] that the semigroup $S_{\epsilon}(t): \mathcal{H}_{j, \epsilon} \rightarrow \mathcal{H}_{j, \epsilon}$ has bounded absorbing sets $\mathcal{B}_{j}$ in $\mathcal{H}_{j, \epsilon}$ of the form

$$
\mathcal{B}_{j}=\left\{(\varphi, \psi, v) \in \mathcal{H}_{j, \epsilon},\|(\varphi, \psi, v)\|_{\mathcal{H}_{j, \epsilon}} \leq r_{j}\right\}, \quad j=1,2,
$$

where $r_{j}>0$ is independent of $\epsilon$. In fact, they are exponentially attracting sets.

\section{Exponential attractors}

Now we state sufficient conditions which guarantee the existence of robust exponential attractors, which are continuous with respect to $\epsilon$ (cf. [2, Theorem 5.1]; also [1, 7, 15]).

Theorem 3.1 ([2]) Let $E^{1}, E^{2}, V^{1}, V^{2}, W^{1}, W^{2}$ be Banach spaces such that $W^{i} \Subset V^{i} \Subset E^{i}$, $i=1,2$. Set $E_{\epsilon}=E^{1} \times E^{2}, V_{\epsilon}=V^{1} \times V^{2}, W_{\epsilon}=W^{1} \times W^{2}$ and endow them with the following norms:

$$
\begin{aligned}
\|(p, q)\|_{E_{\epsilon}} & =\left(\|p\|_{E^{1}}^{2}+\epsilon\|q\|_{E^{2}}^{2}\right)^{1 / 2}, \\
\|(p, q)\|_{V_{\epsilon}} & =\left(\|p\|_{V^{1}}^{2}+\epsilon\|q\|_{V^{2}}^{2}\right)^{1 / 2}, \\
\|(p, q)\|_{W_{\epsilon}} & =\left(\|p\|_{W^{1}}^{2}+\epsilon\|q\|_{W^{2}}^{2}\right)^{1 / 2},
\end{aligned}
$$

respectively, where $\epsilon \in[0,1]$, with the convention that $E_{0}=E^{1}, V_{0}=V^{1}$, and $W_{0}=W^{1}$. Let $B_{\epsilon}(r)$ denote a closed ball in $W_{\epsilon}$ of radius $r>0$ and centered at zero. Consider a oneparameter family of strongly continuous semigroups $\left\{S_{\epsilon}(t)\right\}_{\epsilon}$ acting on the phase-space $E_{\epsilon}$, for each $\epsilon \in[0,1]$. Then assume that there exist $\alpha, \beta, \gamma, \vartheta \in(0,1], \kappa \in\left(0, \frac{1}{2}\right), \Upsilon_{j} \geq 0$, and $\varrho>0$ (all independent of $\epsilon$ ) such that, setting $B_{\epsilon}=B_{\epsilon}(\varrho)$, the following conditions hold:

1. There exists a map $\mathcal{L}: B_{0} \rightarrow V^{2}$ which is Hölder continuous of exponent $\alpha$. Here $B_{0}$ is endowed with the metric topology of $E^{1}$.

2. There exists $t^{\star}>0$, independent of $\epsilon$, such that

$$
S_{\epsilon}(t) B_{\epsilon} \subset B_{\epsilon}, \quad \forall t \geq t^{\star}
$$

and $B_{\epsilon}$ is uniformly bounded (with respect to $\epsilon$ ) in the $E_{1}$-norm. Moreover, setting $S_{\epsilon}\left(t^{\star}\right)=S_{\epsilon}$, the map $S_{\epsilon}$ satisfies, for every $z_{1}, z_{2} \in B_{\epsilon}$,

$$
S_{\epsilon} z_{1}-S_{\epsilon} z_{2}=L_{\epsilon}\left(z_{1}, z_{2}\right)+K_{\epsilon}\left(z_{1}, z_{2}\right)
$$

where

$$
\begin{aligned}
& \left\|L_{\epsilon} z_{1}-L_{\epsilon} z_{2}\right\|_{E_{\epsilon}} \leq \kappa\left\|z_{1}-z_{2}\right\|_{E_{\epsilon}}, \\
& \left\|K_{\epsilon} z_{1}-K_{\epsilon} z_{2}\right\|_{V_{\epsilon}} \leq \Upsilon_{1}\left\|z_{1}-z_{2}\right\|_{E_{\epsilon}} .
\end{aligned}
$$

3. For any $z \in B_{\epsilon}$, there hold

$$
\begin{aligned}
& \left\|S_{\epsilon}^{m} z-\mathcal{L} S_{0}^{m} \Pi_{\epsilon} z\right\|_{E_{1}} \leq \Upsilon_{2}^{m} \epsilon^{\beta}, \quad \forall m \in \mathbb{N}, \\
& \left\|S_{\epsilon}(t) z-\mathcal{L} S_{0}(t) \Pi_{\epsilon} z\right\|_{E_{1}} \leq \Upsilon_{3} \epsilon^{\gamma}, \quad \forall t \in\left[t^{\star}, 2 t^{\star}\right] .
\end{aligned}
$$


Here the "lifting" map $\mathcal{L}: B_{0} \rightarrow E_{\epsilon}$ is defined by

$$
\mathcal{L} x= \begin{cases}(x, \mathcal{L} x), & \text { if } \epsilon>0, \\ x, & \text { if } \epsilon=0,\end{cases}
$$

and $\Pi_{\epsilon}: B_{\epsilon} \rightarrow B_{0}$ is the projection onto the first component when $\epsilon>0$, and the identity map otherwise.

4. The map $z \mapsto S_{\epsilon}(t) z$ is Lipschitz continuous on $B_{\epsilon}$ endowed with the metric topology of $E_{\epsilon}$, with a Lipschitz constant independent of $\epsilon$ and $t \in\left[t^{\star}, 2 t^{\star}\right]$.

5. The map

$$
(t, z) \mapsto S_{\epsilon}(t) z:\left[t^{\star}, 2 t^{\star}\right] \times B_{\epsilon} \rightarrow B_{\epsilon}
$$

is Hölder continuous of exponent $\vartheta$, where $B_{\epsilon}$ is endowed with the metric topology of $E_{\epsilon}$.

Then there exists a family of exponential attractors $\mathcal{E}_{\epsilon}$ on $\mathcal{B}_{\epsilon}=\bar{B}_{\epsilon}{ }^{E_{\epsilon}}$ with the following properties:

(i) $\mathcal{E}_{\epsilon}$ attracts $\mathcal{B}_{\epsilon}$ with an exponential rate which is uniform with respect to $\epsilon$, that is,

$$
\operatorname{dist}_{\epsilon_{\epsilon}}\left(S_{\epsilon}(t) \mathcal{B}_{\epsilon}, \mathcal{E}_{\epsilon}\right) \leq M_{1} e^{-\omega t}, \quad \forall t \geq 0,
$$

for some $M_{1}>0$ and some $\omega>0$.

(ii) The fractal dimension of $\mathcal{E}_{\epsilon}$ (denoted as $\left.\operatorname{dim}_{F}\left(\mathcal{E}_{\epsilon}\right)\right)$ is uniformly bounded with respect to $\epsilon$, that is,

$$
\operatorname{dim}_{F}\left(\mathcal{E}_{\epsilon}\right) \leq M_{2}
$$

(iii) The family $\mathcal{E}_{\epsilon}$ is Hölder continuous with respect to $\epsilon$, that is, there exist a positive constant $M_{3}$ and $\tau \in\left(0, \frac{1}{2}\right]$ such that

$$
\operatorname{dist}_{E_{\epsilon}}^{\mathrm{sym}}\left(\mathcal{E}_{\epsilon}, \mathcal{L} \mathcal{E}_{0}\right) \leq M_{3} \epsilon^{\tau}
$$

for all $0<\epsilon \leq 1$. In addition, there exist a positive constant $M_{4}$ and $\sigma \in\left(0, \frac{1}{2}\right]$ such that

$$
\operatorname{dist}_{E_{1}}\left(\mathcal{E}_{\epsilon}, \mathcal{L} \mathcal{E}_{0}\right) \leq M_{4} \epsilon^{\sigma}
$$

for all $0<\epsilon \leq 1$, and

$$
\lim _{\epsilon \rightarrow 0} \operatorname{dist}_{E_{1}}\left(\mathcal{L} \mathcal{E}_{0}, \mathcal{E}_{\epsilon}\right)=0
$$

Here $\omega, \tau, \sigma$ and $M_{j}$ are independent of $\epsilon$, and they can be computed explicitly.

We observe that the solution to the unperturbed problem (i.e., when $\epsilon=0$ in (1.1)) for the pair $(\phi, u)$ at any time $t$ is given by $(\phi(t), u(t))=S(t)\left(\phi_{0}, u_{0}\right)$ and $\phi_{t}=\mathcal{L}(\phi(t), u(t))$, where

$$
\mathcal{L}(\varphi, \vartheta)=-(-\Delta \varphi+\varphi-g(\varphi)-\vartheta)
$$


Let $z_{1}, z_{2} \in \mathcal{B}_{2}, z_{1}=\left(\phi_{0}^{1}, \phi_{1}^{1}, u_{0}^{1}\right)$ and $z_{2}=\left(\phi_{0}^{2}, \phi_{1}^{2}, u_{0}^{2}\right)$ be initial data for two solutions $\left(\phi^{1}, u^{1}\right)$ and $\left(\phi^{2}, u^{2}\right)$ of $(1.1)$, respectively.

We set $\left(\phi(t), \phi_{t}(t), u(t)\right)=S_{\epsilon}(t) z_{1}-S_{\epsilon}(t) z_{2}, \tilde{\phi}_{0}=\phi_{0}^{1}-\phi_{0}^{2}, \tilde{\phi}_{1}=\phi_{1}^{1}-\phi_{1}^{2}$ and $\tilde{u}_{0}=u_{0}^{1}-u_{0}^{2}$. Furthermore, we perform the splitting

$$
\left(\phi(t), \phi_{t}(t), u(t)\right)=\left(\chi(t), \chi_{t}(t), \vartheta(t)\right)+\left(\Psi(t), \Psi_{t}(t), v(t)\right),
$$

where $K_{\epsilon}\left(z_{1}, z_{2}\right)=\left(\chi(t), \chi_{t}(t), \vartheta(t)\right)$ and $L_{\epsilon}\left(z_{1}, z_{2}\right)=\left(\Psi(t), \Psi_{t}(t), v(t)\right)$ respectively solve the problems:

$$
\left\{\begin{array}{l}
\epsilon \chi_{t t}+\chi_{t}-\Delta \chi_{t}+\chi+g\left(\phi_{1}\right)-g\left(\phi_{2}\right)-\vartheta=0 \\
\vartheta_{t}+\chi_{t}-\Delta \vartheta=0, \\
\left.\chi\right|_{t=0}=0,\left.\quad \chi_{t}\right|_{t=0}=0,\left.\quad \vartheta\right|_{t=0}=0
\end{array}\right.
$$

and

$$
\left\{\begin{array}{l}
\epsilon \Psi_{t t}+\Psi_{t}-\Delta \Psi+\Psi-v=0, \\
v_{t}+\Psi_{t}-\Delta v=0, \\
\left.\Psi\right|_{t=0}=\tilde{\phi}_{0},\left.\quad \Psi_{t}\right|_{t=0}=\tilde{\phi}_{1},\left.\quad v\right|_{t=0}=\tilde{u}_{0} .
\end{array}\right.
$$

Proposition 3.1 There exist $c, c^{\prime}, c_{1}>0$ independent of $\epsilon$ such that

$$
\begin{aligned}
& \left\|L_{\epsilon}\left(z_{1}, z_{2}\right)\right\|_{\mathcal{H}_{1, \epsilon}} \leq c e^{-c_{1} t}\left\|z_{1}-z_{2}\right\|_{\mathcal{H}_{1, \epsilon}}, \quad \forall t \geq 0, \quad \text { and } \\
& \left\|K_{\epsilon}\left(z_{1}, z_{2}\right)\right\|_{\mathcal{H}_{2, \epsilon}} \leq c e^{c^{\prime} t}\left\|z_{1}-z_{2}\right\|_{\mathcal{H}_{1, \epsilon}}^{2}, \quad \forall t \geq 0 .
\end{aligned}
$$

Proof Firstly, we multiply (3.8) $)_{1}$ by $\Psi_{t}$ and $(3.8)_{2}$ by $v$, integrate over $\Omega$, then add the resulting equations to get

$$
\frac{1}{2} \frac{d}{d t}\left(\|\nabla \Psi\|^{2}+\|\Psi\|^{2}+\epsilon\left\|\Psi_{t}\right\|^{2}+\|v\|^{2}\right)+\left\|\Psi_{t}\right\|^{2}+\|\nabla v\|^{2}=0 .
$$

Next, we multiply (3.8) ${ }_{1}$ by $\Psi$ to obtain

$$
\frac{1}{2} \frac{d}{d t}\left[\|\Psi\|^{2}+2 \epsilon\left(\Psi, \Psi_{t}\right)\right]-\epsilon\left\|\Psi_{t}\right\|^{2}+\|\nabla \Psi\|^{2}+\|\Psi\|^{2}-(v, \Psi)=0,
$$

and then deduce that

$$
\frac{1}{2} \frac{d}{d t}\left[\|\Psi\|^{2}+2 \epsilon\left(\Psi, \Psi_{t}\right)\right]+\|\nabla \Psi\|^{2}+\frac{1}{2}\|\Psi\|^{2}+2 \epsilon\left(\Psi_{t}, \Psi\right) \leq 5 \epsilon\left\|\Psi_{t}\right\|^{2}+c\|\nabla v\|^{2} .
$$

Summing (3.11) and $\kappa(3.12)$, for some $\kappa \in(0,1)$ small enough, we get

$$
\begin{aligned}
& \frac{1}{2} \frac{d}{d t}\left(\|\nabla \Psi\|^{2}+(1+\kappa)\|\Psi\|^{2}+\epsilon\left\|\Psi_{t}\right\|^{2}+\|v\|^{2}+2 \kappa \epsilon\left(\Psi, \Psi_{t}\right)\right)+\kappa\|\nabla \Psi\|^{2}+\frac{\kappa}{2}\|\Psi\|^{2} \\
& \quad+\epsilon(1-5 \kappa)\left\|\Psi_{t}\right\|^{2}+(1-c \kappa)\|\nabla v\|^{2}+2 \kappa \epsilon\left(\Psi, \Psi_{t}\right) \leq 0 .
\end{aligned}
$$


Hence, there exists a $c_{1}>0$ (independent of $\epsilon$ ) such that

$$
\frac{d}{d t} E_{2}(t)+c_{1} E_{2}(t) \leq 0
$$

where $E_{2}(t)=\|\nabla \Psi\|^{2}+(1+\kappa)\|\Psi\|^{2}+\epsilon\left\|\Psi_{t}\right\|^{2}+\|v\|^{2}+2 \kappa \epsilon\left(\Psi, \Psi_{t}\right)$. Simple integration over $(0, t)$ gives

$$
E_{2}(t) \leq e^{-c_{1} t} E_{2}(0), \quad \forall t \geq 0
$$

Clearly, by Young's inequality, there exist $b_{3}, b_{4}>0$ (independent of $\epsilon$ ) such that

$$
b_{3}\left\|\left(\Psi, \Psi_{t}, v\right)\right\|_{\mathcal{H}_{1, \epsilon}}^{2} \leq E_{2}(t) \leq b_{4}\left\|\left(\Psi, \Psi_{t}, v\right)\right\|_{\mathcal{H}_{1, \epsilon}}^{2} .
$$

It follows from (3.13) and (3.14) that

$$
\left\|\left(\Psi, \Psi_{t}, v\right)\right\|_{\mathcal{H}_{1, \epsilon}}^{2} \leq e^{-c_{1} t}\left\|\left(\tilde{\phi}_{0}, \tilde{\phi}_{1}, \tilde{u}_{0}\right)\right\|_{\mathcal{H}_{1, \epsilon}}^{2}, \quad \forall t \geq 0 .
$$

Hence (3.9) follows.

Secondly, we multiply $(3.7)_{1}$ by $\chi_{t}$ and $(3.7)_{2}$ by $\vartheta$, integrate over $\Omega$, then add the resulting equations to get

$$
\frac{1}{2} \frac{d}{d t}\left(\|\nabla \chi\|^{2}+\|\chi\|^{2}+\epsilon\left\|\chi_{t}\right\|^{2}+\|\vartheta\|^{2}\right)+\left\|\chi_{t}\right\|^{2}+\|\nabla \vartheta\|^{2}=-\left(g\left(\phi^{1}\right)-g\left(\phi^{2}\right), \chi_{t}\right) .
$$

We have that $\left\|g\left(\phi^{1}\right)-g\left(\phi^{2}\right)\right\| \leq\left\|g^{\prime}\left(\theta \phi^{1}+(1-\theta) \phi^{2}\right)\right\|_{L^{\infty}(\Omega)}\|\phi\|$, where $\theta \in[0,1]$. It follows that

$$
\frac{1}{2} \frac{d}{d t}\left(\|\nabla \chi\|^{2}+\|\chi\|^{2}+\epsilon\left\|\chi_{t}\right\|^{2}+\|\vartheta\|^{2}\right)+\frac{1}{2}\left\|\chi_{t}\right\|^{2}+\|\nabla \vartheta\|^{2} \leq\|\phi\|^{2} .
$$

Integrating (3.15) over $(0, t)$ and then accounting for (2.6), we deduce that

$$
\|\chi\|_{1}^{2}+\epsilon\left\|\chi_{t}\right\|^{2}+\|\vartheta\|^{2} \leq c e^{c^{\prime} t}\left\|\left(\tilde{\phi}_{0}, \tilde{\phi}_{1}, \tilde{u}_{0}\right)\right\|_{\mathcal{H}_{1, \epsilon}}^{2}, \quad \forall t \geq 0 .
$$

Next, we multiply (3.7) by $-\Delta \chi_{t}$ and (3.7) $)_{2}$ by $N \vartheta$, integrate over $\Omega$, then add the resulting equations to get

$$
\begin{aligned}
& \frac{1}{2} \frac{d}{d t}\left(\|\Delta \chi\|^{2}+\|\nabla \chi\|^{2}+\epsilon\left\|\nabla \chi_{t}\right\|^{2}+\|\nabla \vartheta\|^{2}\right)+\left\|\nabla \chi_{t}\right\|^{2}+\|\Delta \vartheta\|^{2} \\
& \quad=-\left(\nabla\left(g\left(\phi^{1}\right)-g\left(\phi^{2}\right)\right), \nabla \chi_{t}\right) .
\end{aligned}
$$

We have that $\left\|\nabla\left(g\left(\phi^{1}\right)-g\left(\phi^{2}\right)\right)\right\| \leq c\|\phi\|_{1}$. It follows that

$$
\frac{1}{2} \frac{d}{d t}\left(\|\Delta \chi\|^{2}+\|\nabla \chi\|^{2}+\epsilon\left\|\nabla \chi_{t}\right\|^{2}+\|\nabla \vartheta\|^{2}\right)+\frac{1}{2}\left\|\nabla \chi_{t}\right\|^{2}+\|\Delta \vartheta\|^{2} \leq c\|\phi\|_{1}^{2} .
$$

Integrating (3.17) over $(0, t)$ and taking into account (2.6), we deduce that

$$
\|\Delta \chi\|^{2}+\|\nabla \chi\|^{2}+\epsilon\left\|\nabla \chi_{t}\right\|^{2}+\|\nabla \vartheta\|^{2} \leq c e^{c^{\prime} t}\left\|\left(\tilde{\phi}_{0}, \tilde{\phi}_{1}, \tilde{u}_{0}\right)\right\|_{\mathcal{H}_{1, \epsilon}}^{2}, \quad \forall t \geq 0 .
$$


On account of (3.16) and (3.18), we obtain that

$$
\left\|\left(\chi, \chi_{t}, \vartheta\right)\right\|_{\mathcal{H}_{2, \epsilon}}^{2} \leq c e^{c^{\prime} t}\left\|\left(\tilde{\phi}_{0}, \tilde{\phi}_{1}, \tilde{u}_{0}\right)\right\|_{\mathcal{H}_{1, \epsilon}}^{2}, \quad \forall t \geq 0
$$

Hence (3.10) follows.

We prove the following result.

Theorem 3.2 For every $\epsilon \in(0,1]$, the semigroup $S_{\epsilon}(t)$ possesses an exponential attractor $\mathcal{E}_{\epsilon}$ (with dimension independent of $\epsilon$ ) in $\mathcal{H}_{1, \epsilon}$.

Proof Let $t \in\left[t^{*}, 2 t^{*}\right]$ and set $\left(\phi(t), \phi_{t}(t), u(u)\right)=S_{\epsilon}(t) z_{01}-S_{\epsilon}(t) z_{02}=\left(\phi^{1}(t), \phi_{t}^{1}(t), u^{1}(t)\right)-$ $\left(\phi^{2}(t), \phi_{t}^{2}(t), u^{2}(t)\right)$. Therefore, the triplet $\left(\phi(t), \phi_{t}(t), u(u)\right)$ is a solution to the problem

$$
\left\{\begin{array}{l}
\epsilon \phi_{t t}+\phi_{t}-\Delta \phi+\phi+g\left(\phi_{1}\right)-g\left(\phi_{2}\right)-u=0, \\
u_{t}+\phi_{t}-\Delta u=0, \\
\left.\phi\right|_{t=0}=\phi^{01}-\phi^{02},\left.\quad \phi_{t}\right|_{t=0}=\phi_{1}^{01}-\phi_{1}^{02},\left.\quad u\right|_{t=0}=u^{01}-u^{02} .
\end{array}\right.
$$

On account of (2.6) we obtain

$$
\left\|S_{\epsilon}(t) z_{01}-S_{\epsilon}(t) z_{02}\right\|_{\mathcal{H}_{1, \epsilon}} \leq c\left(t^{*}\right)\left\|z_{01}-z_{02}\right\|_{\mathcal{H}_{1, \epsilon}}, \quad t \leq 2 t^{*}
$$

where $c\left(t^{*}\right)>0$ is independent of $\epsilon$. Now, we multiply $(1.1)_{1}$ and $(1.1)_{2}$ by $-\Delta \phi_{t}$ and $-\Delta u$, respectively, integrate over $\Omega$ then add the resulting equations, and deduce

$$
\begin{aligned}
& \frac{d}{d t}\left(\|\Delta \phi\|^{2}+\|\nabla \phi\|^{2}+\epsilon\left\|\nabla \phi_{t}\right\|^{2}+\|\nabla u\|^{2}\right)+\left\|\nabla \phi_{t}\right\|^{2}+\|\Delta u\|^{2} \\
& \quad \leq \frac{1}{2}\left\|g^{\prime}(\phi)\right\|_{L^{\infty}(\Omega)}^{2}\|\nabla \phi\|^{2} \\
& \quad \leq c\|\nabla \phi\|^{2} .
\end{aligned}
$$

Integrating over $(0, t)$ and recalling $(2.2)$, we get

$$
\begin{aligned}
& \|\Delta \phi\|^{2}+\|\nabla \phi\|^{2}+\epsilon\left\|\nabla \phi_{t}\right\|^{2}+\|\nabla u\|^{2}+\int_{0}^{t}\left(\left\|\nabla \phi_{t}(s)\right\|^{2}+\|\Delta u(s)\|^{2}\right) d s \\
& \leq c(t+1), \quad \forall t \geq 0 .
\end{aligned}
$$

It then follows from (2.3) and (3.21) that

$$
\int_{0}^{t}\left(\left\|\phi_{t}(s)\right\|_{1}^{2}+\|\Delta u(s)\|^{2}\right) d s \leq c(t+1), \quad \forall t \geq 0
$$

Next, from $(1.1)_{1}$, we deduce that

$$
\epsilon^{2} \int_{0}^{t}\left\|\phi_{t t}(s)\right\|^{2} d s \leq \int_{0}^{t}\left(\left\|\phi_{t}(s)\right\|^{2}+\|\Delta \phi(s)\|^{2}+\|\phi(s)\|^{2}+\|g(\phi(s))\|^{2}+\|u(s)\|^{2}\right) d s
$$


then from (2.2), (3.21) and (3.22) it follows that

$$
\int_{0}^{t} \epsilon\left\|\phi_{t t}(s)\right\|^{2} \leq \frac{c}{\epsilon}(t+1), \quad \forall t \geq 0
$$

Also, from $(1.1)_{2}$ and (3.22), we deduce that

$$
\begin{aligned}
\int_{0}^{t}\left\|u_{t}(s)\right\|^{2} d s & \leq c \int_{0}^{t}\left(\left\|\phi_{t}(s)\right\|^{2}+\|\Delta u(s)\|^{2}\right) d s \\
& \leq c(t+1), \quad \forall t \geq 0 .
\end{aligned}
$$

Finally, we have that

$$
\begin{aligned}
& \left\|S_{\epsilon}(t) z_{01}-S_{\epsilon}\left(t^{\prime}\right) z_{02}\right\|_{\mathcal{H}_{1, \epsilon}} \\
& \quad \leq\left\|S_{\epsilon}(t) z_{01}-S_{\epsilon}\left(t^{\prime}\right) z_{01}\right\|_{\mathcal{H}_{1, \epsilon}}+\left\|S_{\epsilon}\left(t^{\prime}\right) z_{01}-S_{\epsilon}\left(t^{\prime}\right) z_{02}\right\|_{\mathcal{H}_{1, \epsilon}}, \quad \forall t, t^{\prime} \in\left[t^{*}, 2 t^{*}\right] .
\end{aligned}
$$

Indeed, on the one hand, from (3.23) and (3.24), we have

$$
\begin{aligned}
& \left\|S_{\epsilon}(t) z_{01}-S_{\epsilon}\left(t^{\prime}\right) z_{01}\right\|_{\mathcal{H}_{1, \epsilon}} \\
& \quad \leq c\left(\left\|\phi(t)-\phi\left(t^{\prime}\right)\right\|_{1}+\sqrt{\epsilon}\left\|\phi_{t}(t)-\phi_{t}\left(t^{\prime}\right)\right\|+\left\|u(t)-u\left(t^{\prime}\right)\right\|\right) \\
& \quad \leq c \int_{t}^{t^{\prime}}\left(\left\|\phi_{t}(s)\right\|_{1}+\sqrt{\epsilon}\left\|\phi_{t t}(s)\right\|+\left\|u_{t}(s)\right\|\right) d s \\
& \quad \leq c\left(\epsilon, t^{*}\right)\left|t^{\prime}-t\right|^{1 / 2} .
\end{aligned}
$$

On the other hand, it follows from (3.20) that

$$
\left\|S_{\epsilon}\left(t^{\prime}\right) z_{01}-S_{\epsilon}\left(t^{\prime}\right) z_{02}\right\|_{\mathcal{H}_{1, \epsilon}} \leq c\left(t^{*}\right)\left\|z_{01}-z_{02}\right\|_{\mathcal{H}_{1, \epsilon}}, \quad \forall t^{\prime} \geq 0 .
$$

Hence, we conclude with

$$
\left\|S_{\epsilon}(t) z_{01}-S_{\epsilon}\left(t^{\prime}\right) z_{02}\right\|_{\mathcal{H}_{1, \epsilon}} \leq c\left(\epsilon, t^{*}\right)\left(\left|t^{\prime}-t\right|^{1 / 2}+\left\|z_{01}-z_{02}\right\|_{\mathcal{H}_{1, \epsilon}}\right)
$$

We now apply Theorem 3.1. We will only need to check Assumptions 2, 4 and 5, for the existence of a family of exponential attractors $\mathcal{E}_{\epsilon}$ that satisfy (3.1) and (3.2). Assumption 2 follows from estimates (3.9) and (3.10) of Proposition 3.1. Assumptions 4 and 5 follow from (2.6) and (3.26), respectively. This shows the existence of a family of exponential attractors $\mathcal{E}_{\epsilon}$ in $\mathcal{H}_{1, \epsilon}$ with dimension independent of $\epsilon$.

\section{Robust family of exponential attractors}

We start by showing the existence of an absorbing set in $\mathcal{H}_{3, \epsilon}$.

Proposition 4.1 The semigroup $S_{\epsilon}(t)$ possesses an exponentially attracting bounded absorbing set $\mathcal{B}_{3}$ in $\mathcal{H}_{3, \epsilon}$. 
Proof Let $B \subset \mathcal{H}_{3, \epsilon}$ be a bounded set, and let $\left(\phi_{0}, \phi_{1}, u_{0}\right) \in B$. Hence, since $\mathcal{H}_{3, \epsilon} \subset \mathcal{H}_{2, \epsilon}$, there exists a $t(B)>0$ such that $\left(\phi(t), \phi_{t}(t), u(t)\right) \in \mathcal{B}_{2}, \forall t \geq t(B)$. That is,

$$
\|\phi(t)\|_{2}^{2}+\epsilon\left\|\phi_{t}(t)\right\|_{1}^{2}+\|u(t)\|_{1}^{2} \leq r_{2}, \quad \forall t \geq t(B)
$$

The following estimates hold true:

$$
\begin{aligned}
\left(\Delta g(\phi), \Delta \phi_{t}\right) & \leq\left\|g^{\prime}(\phi)\right\|_{L^{\infty}(\Omega)}\|\Delta \phi\|\left\|\Delta \phi_{t}\right\|+\left\|g^{\prime \prime}(\phi)\right\|_{L^{\infty}(\Omega)}\|\nabla \phi\|_{L^{4} \Omega}^{2}\left\|\Delta \phi_{t}\right\| \\
& \leq c\left(\left\|g^{\prime}(\phi)\right\|_{L^{\infty}(\Omega)}^{2}\|\Delta \phi\|^{2}+\left\|g^{\prime \prime}(\phi)\right\|_{L^{\infty}(\Omega)}^{2}\|\nabla \phi\|_{1}^{4}\right)+\frac{1}{2}\left\|\Delta \phi_{t}\right\|^{2}, \\
\left(g(\phi), \Delta^{2} \phi\right) \leq & \left\|g^{\prime}(\phi)\right\|_{L^{\infty}(\Omega)}\|\nabla \phi\|\|\nabla \Delta \phi\| \\
\leq & \left\|g^{\prime}(\phi)\right\|_{L^{\infty}(\Omega)}^{2}\|\nabla \phi\|^{2}+\frac{1}{4}\|\nabla \Delta \phi\|^{2}, \\
\left(u, \Delta^{2} \phi\right) \leq\|\nabla u\|^{2}+\frac{1}{4}\|\nabla \Delta \phi\|^{2}, &
\end{aligned}
$$

Multiply (1.1) by $\Delta^{2} \phi_{t}$ and $\kappa \Delta^{2} \phi$ with $0<\kappa \leq \frac{1}{8}$, then multiply $(1.1)_{2}$ by $\Delta^{2} u$, and integrate over $\Omega$. Adding the resulting equations gives, on account of (4.2)-(4.5),

$$
\begin{gathered}
\frac{1}{2} \frac{d}{d t}\left[\|\nabla \Delta \phi\|^{2}+(1+\kappa)\|\Delta \phi\|^{2}+\epsilon\left\|\Delta \phi_{t}\right\|^{2}+\|\Delta u\|^{2}+2 \kappa \epsilon\left(\Delta \phi, \Delta \phi_{t}\right)\right] \\
+\frac{\kappa}{2}\left\|\left.\nabla \Delta \phi\right|^{2}+\frac{\kappa}{2}\right\| \Delta \phi\left\|^{2}+\epsilon\left(\frac{1}{2}-2 \kappa\right)\right\| \Delta \phi_{t} \|^{2}+\epsilon \kappa\left(\Delta \phi, \Delta \phi_{t}\right) \\
\leq c\left(\left\|g^{\prime}(\phi)\right\|_{L^{\infty}(\Omega)}^{2}\|\Delta \phi\|^{2}+\left\|g^{\prime \prime}(\phi)\right\|_{L^{\infty}(\Omega)}^{2}\|\nabla \phi\|_{1}^{4}+\|\nabla u\|^{2}\right) .
\end{gathered}
$$

Hence from (4.1), there exists a constant $\varpi_{1}>0$ independent of $\epsilon$ such that

$$
\frac{d}{d t} E_{3}(t)+\varpi_{1} E_{3}(t) \leq c\left(r_{2}\right)
$$

where

$$
E_{3}(t)=\|\nabla \Delta \phi\|^{2}+(1+\varpi)\|\Delta \phi\|^{2}+\epsilon\left\|\Delta \phi_{t}\right\|^{2}+\|\Delta u\|^{2}+2 \varpi \epsilon\left(\Delta \phi, \Delta \phi_{t}\right) .
$$

Clearly, by Hölder's and Young's inequalities, there exist constants $\varpi_{2}, \varpi_{3}>0$, independent of $\epsilon$ such that

$$
\begin{aligned}
& \varpi_{2}\left(\|\nabla \Delta \phi\|^{2}+\|\Delta \phi\|^{2}+\epsilon\left\|\Delta \phi_{t}\right\|^{2}+\|\Delta u\|^{2}\right) \\
& \quad \leq E_{3}(t) \\
& \quad \leq \varpi_{3}\left(\|\nabla \Delta \phi\|^{2}+\|\Delta \phi\|^{2}+\epsilon\left\|\Delta \phi_{t}\right\|^{2}+\|\Delta u\|^{2}\right) .
\end{aligned}
$$

Applying the generalized Gronwall's lemma to (4.6) and using (4.7), we obtain

$$
\left\|\left(\phi(t), \phi_{t}(t), u(t)\right)\right\|_{\mathcal{H}_{3, \epsilon}}^{2} \leq c(B) e^{-\varpi_{1} t}+c\left(r_{2}\right), \quad \forall t \geq 0 .
$$


Hence, we have that

$$
\mathcal{B}_{3}=\left\{(\varphi, \psi, v) \in \mathcal{H}_{3, \epsilon},\|(\varphi, \psi, v)\|_{\mathcal{H}_{3, \epsilon}} \leq \sqrt{2 c\left(r_{2}\right) / \varpi_{1}}=r_{3}\right\}
$$

is an exponentially attracting absorbing set for $S_{\epsilon}(t)$ on $\mathcal{H}_{3 ., \epsilon}$.

We prove the following result.

Proposition 4.2 For every $\epsilon \in(0,1]$, there exists a $c>0$, independent of $\epsilon$, such that for any $\mathrm{z} \in \mathcal{B}_{3}$,

$$
\left\|S_{\epsilon}(t) \mathrm{z}\right\|_{\mathcal{H}_{2,0}} \leq c, \quad \forall t \geq 1
$$

Proof Let $\mathrm{z}_{0}=\left(\phi_{0}, \phi_{1}, u_{0}\right) \in \mathcal{B}_{3}$. We set $\left(\phi(t), \phi_{t}(t), u(t)\right)=S_{\epsilon}(t)\left(\phi_{0}, \phi_{1}, u_{0}\right), \forall t \geq 0$. There exists a $c>0$, independent of $\epsilon$, such that

$$
\|\phi(t)\|_{3}^{2}+\epsilon\left\|\phi_{t}(t)\right\|_{2}^{2}+\|u(t)\|_{2}^{2} \leq c, \quad \forall t \geq 0
$$

Multiplying the first equation of (1.1) by $\Gamma \phi_{t}$, where $\Gamma=I-\Delta$, then integrating over $\Omega$, we obtain

$$
\frac{\epsilon}{2} \frac{d}{d t}\left\|\phi_{t}\right\|_{1}^{2}+\left\|\phi_{t}\right\|_{1}^{2}+\left(-\Delta \phi, \Gamma \phi_{t}\right)+\left(\phi, \Gamma \phi_{t}\right)+\left(g(\phi), \Gamma \phi_{t}\right)-\left(u, \Gamma \phi_{t}\right)=0 .
$$

Hence, we deduce due to (4.10), that

$$
\epsilon \frac{d}{d t}\left\|\phi_{t}\right\|_{1}^{2}+\left\|\phi_{t}\right\|_{1}^{2} \leq c
$$

First, we multiply (4.11) by $e^{c t / \epsilon}$ and integrate between $\tau$ and $t+1$, for any $\tau \leq t+1$. This yields

$$
\epsilon\left\|\phi_{t}(t+1)\right\|_{1}^{2} e^{c(t+1) / \epsilon} \leq c \epsilon\left\|\phi_{t}(\tau)\right\|_{1}^{2} e^{c s / \epsilon}+c \epsilon\left(e^{c(t+1) / \epsilon}-e^{c \tau / \epsilon}\right) .
$$

Now, integrating (4.12) between $t$ and $t+1$ with respect to $\tau$, we deduce

$$
\left\|\phi_{t}(t)\right\|_{1}^{2} \leq c, \quad \forall t \geq 1
$$

hence the estimate (4.9) holds.

The following estimate holds for difference of two solutions.

Proposition 4.3 There exist $t_{\star}>0, c$ and $c^{\prime}>0$ all independent of $\epsilon$ such that

$$
\left\|S_{\epsilon}(t)\left(\phi_{0}, \phi_{1}, u_{0}\right)-\mathcal{L} S(t)\left(\phi_{0}, u_{0}\right)\right\|_{\mathcal{H}_{1, \epsilon}}^{2} \leq c \sqrt[4]{\epsilon} e^{c^{\prime} t}, \quad \forall t \geq t_{\star}
$$

for any $\left(\phi_{0}, \phi_{1}, u_{0}\right) \in \mathcal{B}_{3}$, and

$$
\left\|S_{\epsilon}(t)\left(\phi_{0}, \phi_{1}, u_{0}\right)-\mathcal{L} S(t)\left(\phi_{0}, u_{0}\right)\right\|_{\mathcal{H}_{1,0}}^{2} \leq c \sqrt[4]{\epsilon} e^{c^{\prime} t}, \quad \forall t \geq t_{\star},
$$


for any $\left(\phi_{0}, \phi_{1}, u_{0}\right) \in S_{\epsilon}(1) \mathcal{B}_{3}$, and any $\epsilon \in(0,1]$, where $\mathcal{L}(\psi(t), v(t))=(\psi(t), \mathcal{L}(\psi(t)$, $v(t)), v(t))$.

Proof Let $\left(\phi_{0}, \phi_{1}, u_{0}\right) \in \mathcal{B}_{3}$. We set $\left(\phi^{\epsilon}(t), \phi_{t}^{\epsilon}(t), u^{\epsilon}(t)\right)=S_{\epsilon}(t)\left(\phi_{0}, \phi_{1}, u_{0}\right)$, and $\left(\phi(t), \phi_{t}(t)\right.$, $u(t))=\mathcal{L} S(t)\left(\phi_{0}, u_{0}\right)$.

We have that

$$
\begin{aligned}
& \left\|\phi^{\epsilon}(t)\right\|_{3}^{2}+\epsilon\left\|\phi_{t}^{\epsilon}(t)\right\|_{2}^{2}+\left\|u^{\epsilon}(t)\right\|_{2}^{2} \leq c, \quad \forall t \geq 0, \\
& \|\phi(t)\|_{3}^{2}+\|u(t)\|_{2}^{2} \leq c, \quad \forall t \geq 0 .
\end{aligned}
$$

We set $P=\phi^{\epsilon}-\phi$ and $R=u^{\epsilon}-u$, then the pair $(P, R)$ solves the problem:

$$
\left\{\begin{array}{l}
\epsilon P_{t t}+P_{t}-\Delta P+P+g\left(\phi^{\epsilon}\right)-g(\phi)-R=-\epsilon \phi_{t t}, \\
R_{t}+P_{t}-\Delta R=0, \\
\left.P\right|_{t=0}=0,\left.\quad P_{t}\right|_{t=0}=\phi_{1}-\mathcal{L}\left(\phi_{0}, u_{0}\right),\left.\quad R\right|_{t=0}=0 .
\end{array}\right.
$$

We multiply (4.18) ${ }_{1}$ and $(4.18)_{1}$ by $P_{t}$ and $R$, respectively, then integrate over $\Omega$. Adding the resulting equations, we obtain

$$
\frac{1}{2} \frac{d}{d t}\left(\|P\|_{1}^{2}+\epsilon\left\|P_{t}\right\|^{2}+\|R\|^{2}\right)+\left\|P_{t}\right\|+\|\nabla R\|^{2}=-\left(g\left(\phi^{\epsilon}\right)-g(\phi), P_{t}\right)-\epsilon\left(\phi_{t t}, P_{t}\right) .
$$

We deduce that

$$
\frac{d}{d t}\left(\|P\|_{1}^{2}+\epsilon\left\|P_{t}\right\|^{2}+\|R\|^{2}\right)+\left\|P_{t}\right\|^{2}+\|\nabla R\|^{2} \leq c^{\prime}\|P\|^{2}+2 \epsilon^{2}\left\|\phi_{t t}\right\|^{2} .
$$

The following holds true:

$$
\int_{0}^{t}\left\|\phi_{t t}(s)\right\|^{2} d s \leq c e^{\nu t}, \quad \forall t \geq 0
$$

We integrate (4.19) over $(0, t)$, and on account of (4.20) we obtain

$$
\|P(t)\|_{1}^{2}+\epsilon\left\|P_{t}(t)\right\|^{2}+\|R(t)\|^{2} \leq c\left(\epsilon\left\|\phi_{1}-\mathcal{L}\left(\phi_{0}, u_{0}\right)\right\|^{2}+\epsilon^{2}\right) e^{c^{\prime} t}, \quad \forall t \geq 0
$$

Similarly, we multiply $(4.18)_{1}$ and $(4.18)_{1}$ by $-\Delta P_{t}$ and $-\Delta R$, respectively, then integrate over $\Omega$. Adding the resulting equations and proceeding like in the proof of estimate (4.21) above, we obtain

$$
\|P(t)\|_{2}^{2}+\epsilon\left\|\nabla P_{t}(t)\right\|^{2}+\|R(t)\|_{1}^{2} \leq c\left(\epsilon\left\|\phi_{1}-\mathcal{L}\left(\phi_{0}, u_{0}\right)\right\|_{1}^{2}+\epsilon^{2}\right) e^{c^{\prime} t}, \quad \forall t \geq 0 .
$$

Now, integrating (4.19) between 0 and $t$, we obtain

$$
\int_{0}^{t}\left(\left\|P_{t}(s)\right\|^{2}+\|R(s)\|_{1}^{2}\right) d s \leq c\left(\epsilon\left\|\phi_{1}-\mathcal{L}\left(\phi_{0}, u_{0}\right)\right\|^{2}+\epsilon^{2}\right) e^{c^{\prime} t}, \quad \forall t \geq 0,
$$


due to (4.20) and (4.21). Next, we multiply (4.18) $)_{1}$ by $P_{t}$ and integrate over $\Omega$ to deduce

$$
\frac{d}{d t} \epsilon\left\|P_{t}\right\|^{2}+\left\|P_{t}\right\|^{2} \leq c\left(\|P\|_{2}^{2}+\|R\|^{2}+\epsilon^{2}\left\|\phi_{t t}\right\|^{2}\right) .
$$

We multiply (4.24) by $t$ to get

$$
\frac{d}{d t}\left(\epsilon t\left\|P_{t}\right\|^{2} e^{t / \epsilon}\right) \leq \epsilon\left\|P_{t}\right\|^{2} e^{t / \epsilon}+\left[c t\left(\|P\|^{2}+\|R\|^{2}+\epsilon^{2}\left\|\phi_{t t}\right\|^{2}\right)\right] e^{t / \epsilon} .
$$

Integrating (4.25) between 0 and $t$, due to (4.20), (4.21), (4.22) and (4.23), we obtain

$$
\begin{aligned}
\epsilon t\left\|P_{t}(t)\right\|^{2} \leq & \epsilon \int_{0}^{t}\left\|P_{t}(s)\right\|^{2} d s+c \epsilon t\left(\epsilon\left\|\phi_{1}-\mathcal{L}\left(\phi_{0}, u_{0}\right)\right\|_{1}^{2}+\epsilon^{2}\right) e^{c^{\prime} t} \\
& +c \epsilon^{2} t \int_{0}^{t}\left\|\phi_{t t}(s)\right\|^{2} d s \\
\leq & c \epsilon\left(\epsilon^{2}+\epsilon\left\|\phi_{1}-\mathcal{L}\left(\phi_{0}, u_{0}\right)\right\|^{2}\right) e^{c^{\prime} t}+c t \epsilon\left(\epsilon^{2}+\epsilon\left\|\phi_{1}-\mathcal{L}\left(\phi_{0}, u_{0}\right)\right\|_{1}^{2}\right) e^{c^{\prime} t} \\
& +c t \epsilon^{2} e^{c^{\prime} t}, \quad \forall t \geq 0 .
\end{aligned}
$$

Hence

$$
\begin{aligned}
\epsilon\left\|P_{t}(t)\right\|^{2} \leq & c \epsilon t^{-1}\left(\epsilon^{2}+\epsilon\left\|\phi_{1}-\mathcal{L}\left(\phi_{0}, u_{0}\right)\right\|^{2}\right) e^{c^{\prime} t} \\
& +c \epsilon\left(\epsilon+\epsilon\left\|\phi_{1}-\mathcal{L}\left(\phi_{0}, u_{0}\right)\right\|_{1}^{2}\right) e^{c^{\prime} t}, \quad \forall t \geq 0 .
\end{aligned}
$$

Therefore, we have

$$
\epsilon\left\|P_{t}(\sqrt{\epsilon})\right\|^{2} \leq c \sqrt{\epsilon}\left(\epsilon^{2}+\epsilon\left\|\phi_{1}-\mathcal{L}\left(\phi_{0}, u_{0}\right)\right\|^{2}\right)+c \epsilon\left(\epsilon+\epsilon\left\|\phi_{1}-\mathcal{L}\left(\phi_{0}, u_{0}\right)\right\|_{1}^{2}\right) .
$$

Using the interpolation inequality, (4.22) and (4.23), we deduce

$$
\begin{aligned}
\|P(t)\|_{1}^{2} & \leq c\|P(t)\|\|P(t)\|_{2} \\
& \leq c \sqrt{t}\left(\epsilon^{2}+\epsilon\left\|\phi_{1}-\mathcal{L}\left(\phi_{0}, u_{0}\right)\right\|_{1}^{2}\right) e^{c^{\prime} t}, \quad \forall t \geq 0 .
\end{aligned}
$$

Therefore,

$$
\|P(\sqrt{\epsilon})\|_{1}^{2} \leq c \sqrt[4]{\epsilon}\left(\epsilon^{2}+\epsilon\left\|\phi_{1}-\mathcal{L}\left(\phi_{0}, u_{0}\right)\right\|_{1}^{2}\right) .
$$

From (4.18) $)_{2}$ and (4.23), we deduce

$$
\begin{aligned}
\int_{0}^{t}\left\|R_{t}(s)\right\|_{-1}^{2} d s & \leq c \int_{0}^{t}\left(\left\|P_{t}(s)\right\|^{2}+\|\nabla R(s)\|^{2}\right) d s \\
& \leq c\left(\epsilon^{2}+\epsilon\left\|\phi_{1}-\mathcal{L}\left(\phi_{0}, u_{0}\right)\right\|^{2}\right) e^{c^{\prime} t}, \quad \forall t \geq 0 .
\end{aligned}
$$

Again, by interpolation inequality, (4.22) and (4.28), we have

$$
\begin{aligned}
\|R(t)\|^{2} & \leq c\|R(t)\|_{-1}\|R(t)\|_{1} \\
& \leq c \sqrt{t}\left(\epsilon^{2}+\epsilon\left\|\phi_{1}-\mathcal{L}\left(\phi_{0}, u_{0}\right)\right\|_{1}^{2}\right) e^{c^{\prime} t}, \quad \forall t \geq 0,
\end{aligned}
$$


so that

$$
\|R(\sqrt{\epsilon})\|^{2} \leq c \sqrt[4]{\epsilon}\left(\epsilon^{2}+\epsilon\left\|\phi_{1}-\mathcal{L}\left(\phi_{0}, u_{0}\right)\right\|_{1}^{2}\right) .
$$

We now apply Gronwall's lemma to (4.19) between $\sqrt{\epsilon}$ and $t+\sqrt{\epsilon}$. We find

$$
\left(\|P\|_{1}^{2}+\epsilon\left\|P_{t}\right\|^{2}+\|R\|^{2}\right)(t+\sqrt{\epsilon}) \leq c\left[\left(\|P\|_{1}^{2}+\epsilon\left\|P_{t}\right\|^{2}+\|R\|^{2}\right)(\sqrt{\epsilon})+\epsilon^{2}\right] e^{c^{\prime} t},
$$

for every $t \geq 0$.

Due to (4.26), (4.27) and (4.29), from (4.30) it follows that

$$
\left(\|P\|_{1}^{2}+\epsilon\left\|P_{t}\right\|^{2}+\|R\|^{2}\right)(t+\sqrt{\epsilon}) \leq c \sqrt[4]{\epsilon}\left(\epsilon+\epsilon\left\|\phi_{1}-\mathcal{L}\left(\phi_{0}, u_{0}\right)\right\|_{1}^{2}\right) e^{c^{\prime} t}, \quad \forall t \geq 0 .
$$

Again, integrating (4.19) between $s$ and $t$, we arrive at the following estimate:

$$
\|P(t)\|_{1}^{2}+\epsilon\left\|P_{t}(t)\right\|^{2}+\|R(t)\|^{2} \leq c\left(\|P(s)\|_{1}^{2}+\epsilon\left\|P_{t}(s)\right\|^{2}+\|R(s)\|^{2}+\epsilon^{2}\right) e^{c^{\prime} t},
$$

for any given $s \geq 0$ and any $t>s$. Let $t_{\star}>0$, independent of $\epsilon$, be such that $t_{\star}>\sqrt{\epsilon}$. This latter estimate, with $s=\sqrt{\epsilon}$, in combination with (4.31) gives

$$
\|P(t)\|_{1}^{2}+\epsilon\left\|P_{t}(t)\right\|^{2}+\|R(t)\|^{2} \leq c \sqrt[4]{\epsilon}\left(\epsilon+\epsilon\left\|\phi_{1}-\mathscr{L}\left(\phi_{0}, u_{0}\right)\right\|_{1}^{2}\right) e^{c^{\prime} t}, \quad \forall t>\sqrt{\epsilon} .
$$

Finally, estimate (4.14) follows from (4.32) while estimate (4.15) follows from (4.9) and (4.32).

We have the following corollary of Proposition 4.3.

\section{Corollary 4.1}

$$
\left\|\Pi_{\epsilon} S_{\epsilon}(t)\left(\phi_{0}, \phi_{1}, u_{0}\right)-S(t)\left(\phi_{0}, u_{0}\right)\right\|_{\mathcal{H}_{1,0}}^{2} \leq c \sqrt[4]{\epsilon} e^{c^{\prime} t}, \quad \forall t \geq t_{\star},
$$

where $\Pi_{\epsilon}(X \times Y \times Z)=X \times Z$, i.e., $\left\|\phi^{\epsilon}(t)-\phi(t)\right\|_{1}^{2}+\left\|u^{\epsilon}(t)-u(t)\right\|^{2} \leq c \sqrt[4]{\epsilon} e^{c^{\prime} t}, \forall t \geq t_{\star}$.

The semigroup $S(t)$ for the variable $(\phi, u)$ alone possesses an exponential attractor $\mathcal{E}_{0}$ on $\mathcal{H}_{1,0}$, see Theorem 9.14 in [11]. We set $\widetilde{\mathcal{B}}_{3}=S_{\epsilon}\left(t^{*}\right) \mathcal{B}_{3}$, where $t^{*}>0$ is independent of $\epsilon$.

Theorem 4.1 There exist $\varpi_{1}, \varpi_{2} \in\left(0, \frac{1}{2}\right]$ and $M_{1}, M_{2}>0$, all independent of $\epsilon$, and a family of exponential attractors $\mathcal{E}_{\epsilon}$ enjoying all the properties of Theorem 3.2 and such that

$$
\begin{aligned}
& \operatorname{dist}_{\mathcal{H}_{1, \epsilon}}^{\text {sym }}\left(\mathcal{E}_{\epsilon}, \mathcal{E}\right) \leq M_{1} \epsilon^{\varpi_{1}}, \\
& \operatorname{dist}_{\mathcal{H}_{1,0}}\left(\mathcal{E}_{\epsilon}, \mathcal{E}\right) \leq M_{2} \varepsilon^{\varpi_{2}}, \quad \text { and } \\
& \lim _{\epsilon \rightarrow 0} \operatorname{dist}_{\mathcal{H}_{1,0}}\left(\mathcal{E}, \mathcal{E}_{\epsilon}\right)=0,
\end{aligned}
$$

where $\mathcal{E}=\mathcal{L E}_{0}=\left\{(\varphi, \mathcal{L}(\varphi, \vartheta), \vartheta),(\varphi, \vartheta) \in \mathcal{E}_{0}\right\}$ 
Proof On account of Theorem 3.1, we let $E_{\epsilon}=\mathcal{H}_{1, \epsilon}, V_{\epsilon}=\mathcal{H}_{2, \epsilon}, W_{\epsilon}=\mathcal{H}_{3, \epsilon}, B_{\epsilon}=\widetilde{\mathcal{B}}_{4}$ and we check all Assumptions 1-5. To verify Assumption 1, using the interpolation inequality, there exists a constant $c$ such that for some $\theta \in[0,1]$ we have

$$
\begin{aligned}
\|\mathcal{L}(\varphi, \vartheta)-\mathcal{L}(\psi, v)\| & \leq\|\Delta(\varphi-\psi)\|+\|\varphi-\psi\|+\|g(\varphi)-g(\psi)\|+\|\vartheta-v\| \\
& \leq c\left(\|\varphi-\psi\|^{1 / 2}+\|\varphi-\psi\|_{3}^{1 / 2}\right)\|\varphi-\psi\|_{1}^{1 / 2}+\|\vartheta-v\| \\
& \leq c\left(\|\varphi-\psi\|_{1}^{1 / 2}+\|\vartheta-v\|^{1 / 2}\right)
\end{aligned}
$$

for any $(\varphi, \vartheta)$ and $(\psi, \nu)$ in $\mathcal{B}$.

Assumptions 2, 4 and 5 were checked in Theorem 3.2. Assumption 3 follows from (4.14) and (4.15). This shows the existence of exponential attractors in $\mathcal{H}_{1,0}$ that satisfy (4.34), (4.35) and (4.36).

We also state the following theorem, which is a direct consequence of Corollary 4.33.

Theorem 4.2 For every $\epsilon \in(0,1]$, there exists a constant $M_{1}>0$ independent of $\epsilon$ such that the family of exponential attractors $\mathcal{E}_{\epsilon}$ of the semigroup $S_{\epsilon}(t)$ on $\mathcal{H}_{1, \epsilon}$ satisfies

$$
\operatorname{dist}_{\mathcal{H}_{1,0}}^{\text {sym }}\left(\Pi_{\epsilon} \mathcal{E}_{\epsilon}, \mathcal{E}_{0}\right) \leq M_{1} \sqrt[4]{\epsilon}
$$

\section{Conclusion}

In this work, we considered a parabolic-hyperbolic phase-field system, a model which describes phase separation in material sciences. An example is melting and solidification processes. We constructed a robust family of exponential attractors, which are both upper and lower semicontinuous at the parameter $\epsilon=0$. A consequence of this is the existence of fractal dimensional global attractor and, moreover, the dynamics of the global attractor converges to that of the well known Cagilnap phase-field system. Most interestingly, estimates were obtained in norms which are independent of the parameter $\epsilon$.

\section{Acknowledgements}

The author is grateful to University of Hafr Al Batin for her continuous support.

\section{Funding}

Not applicable.

Abbreviations

PHPFS, Parabolic-Hyperbolic Phase-Field System.

Availability of data and materials

Not applicable.

Competing interests

The author declares that there is no competing interest.

Authors' contributions

The author read and approved the final manuscript.

\section{Publisher's Note}

Springer Nature remains neutral with regard to jurisdictional claims in published maps and institutional affiliations. 
References

1. Bonfoh, A., Enyi, C.D.: Large time behavior of a conserved phase-field system. Commun. Pure Appl. Anal. 15 1077-1105 (2016)

2. Bonfoh, A., Grasselli, M., Miranville, A.: Singularly perturbed 1D Cahn-Hilliard equation revisited. Nonlinear Differ. Equ. Appl. 17, 663-695 (2010)

3. Bonfoh, A., Miranville, A.: On Cahn-Hilliard-Gurtin equations. Nonlinear Anal. 47, 3455-3466 (2001)

4. Calgaro, C., Goubet, O., Zahrouni, E.: Finite-dimensional global attractor for a semi-discrete fractional nonlinear Schrödinger equation. Math. Methods Appl. Sci. 40(15), 5563-5574 (2017)

5. Dung, L., Nicolaenko, B.: Exponential attractors in Banach spaces. J. Dyn. Differ. Equ. 13, $791-806$ (2001)

6. Eden, A., Foias, C., Nicolaenko, B., Temam, R.: Exponential Attractors for Dissipative Evolution Equations. Research in Applied Mathematics, vol. 37. Wiley, New York (1994)

7. Gatti, S., Grasselli, M., Miranville, A., Pata, V.: A construction of a robust family of exponential attractors. Proc. Am. Math. Soc. 134, 117-127 (2006)

8. Goubet, O., Zahrouni, E.: Finite dimensional global attractor for a fractional nonlinear Schrödinger equation. NoDEA Nonlinear Differ. Equ. Appl. 24(5), Article ID 59 (2017)

9. Grasselli, M., Miranville, A., Pata, V., Zelik, S.: Well-posedness and long time behavior of a parabolic-hyperbolic phase-field system with singular potentials. Math. Nachr. 280, 1475-1509 (2007)

10. Grasselli, M., Pata, V.: Existence of a universal attractor for a parabolic-hyperbolic phase-field system. Adv. Math. Sci. Appl. 13, 443-459 (2003)

11. Grasselli, M., Pata, V.: Asymptotic behavior of a parabolic-hyperbolic system. Commun. Pure Appl. Anal. 3, 849-881 (2004)

12. Grasselli, M., Petzeltová, H., Schimperna, G.: Convergence to stationary solutions for a parabolic-hyperbolic phase-field system. Commun. Pure Appl. Anal. 5, 827-838 (2006)

13. Jung, C.Y.,Park, E., Temam, R.: Boundary layer analysis of nonlinear reaction-diffusion equations in a smooth domain. Adv. Nonlinear Anal. 6(3), 277-300 (2017)

14. Miranville, A.: Exponential attractors for a class of evolution equations by a decomposition method. C. R. Acad. Sci., Sér. 1 Math. 328, 145-150 (1999)

15. Miranville, A., Zelik, S.: Attractors for dissipative partial differential equations in bounded and unbounded domains. In: Handbook of Differential Equations: Evolutionary Equations, vol. IV, pp. 103-200. Elsevier, Amsterdam (2008)

16. Wu, H., Grasselli, M., Zheng, S.: Convergence to equilibrium for a parabolic-hyperbolic phase-field system with dynamical boundary condition. J. Math. Anal. Appl. 329, 948-976 (2007)

17. Wu, H., Grasselli, M., Zheng, S.: Convergence to equilibrium for a parabolic-hyperbolic phase-field system with Neumann boundary conditions. Math. Models Methods Appl. Sci. 17, 125-153 (2007)

\section{Submit your manuscript to a SpringerOpen ${ }^{\circ}$ journal and benefit from:}

- Convenient online submission

- Rigorous peer review

- Open access: articles freely available online

- High visibility within the field

Retaining the copyright to your article

Submit your next manuscript at $>$ springeropen.com 